\title{
Modeling and Implementing Nonlinear Equations in Solid-State Lasers for Studying their Performance
}

\author{
Ali Roudehghat Shotorbani ${ }^{a}$, Hassan Rasooli Saghai ${ }^{\text {* }}$ \\ ${ }^{a}$ Department of Electrical Engineering, Faculty of Engineering, Islamic Azad University, Tabriz Branch, Tabriz, Iran \\ ${ }^{b}$ Associate Professor of Electrical Engineering, Islamic Azad University, Tabriz Branch, Tabriz, Iran
}

\begin{abstract}
In this paper, the effect of radius variation of beam light on output efficacy of SFD Yttrium aluminium borate laser doped with Neodymium ion, which is simultaneously a non-linear and active laser crystal, is investigated in a double-pass cavity. This is done with a concave lens that concentrates (Reduction of optical radius within nonlinear material) as much optical laser as possible, resulting in increasing the laser efficiency, second harmonic and the population inversion difference. In this study, we first developed five discrete differential equations describing the interactions of $807 \mathrm{~nm}$ pump beam, $1060 \mathrm{~nm}$ laser beam and 530nm second harmonic beam. Output efficiencies of laser and second harmonic beams at pumping power of $\mathrm{Pp}=20 \mathrm{~W}$ and beam radius of $5 \mu \mathrm{m}$ have been presented. Meanwhile, in this paper, the first experiment for creating second harmonic in solid state lasers was fully described with a figure and its procedure was investigated and then the equations (second harmonic and laser and population inversion) were studied. Radius variation of beam light aims at increasing laser output efficacy and improving second harmonic and population inversion. The analytic methods which have been solved the discrete differential equations via Matlab.
\end{abstract}

\section{Keywords:}

Yttrium Aluminum Borate Laser Non-Linear and Active Laser Crystal; Pump;

Neodymium Ion;

Beam Radius.

\section{Article History:}

Received: 17 November 2017

Accepted: 15 March 2018

\section{1- Introduction}

Having used non-linear optical materials like harmonic producers and optical parameters oscillators, we can develop the frequency area in accessible lasers well. A laser spring with high power and non-linear materials can make the frequency of radiation laser as twice, three times or four times, non-linear crystals which are used for producing manifold frequencies compared to primary frequency provides the reproduction of frequency in a KP*D non-linear crystal via a proper laser having high energy photons like $\mathrm{Nd}$ :YAB laser [1].

Jaque in 2001 could produce three main colors of red, blue and green in NYAB crystal using some of non-linear phenomena such as the combination of self-accumulation frequency [5].

Jaque et.al in 2004 studied the effect of $+\mathrm{Nd} 3$ and $+\mathrm{Yb} 3$ on energy conversion output in $\mathrm{YAB}$ crystal and determined optimal value for laser usages [3].

Having published papers during 2009 to 2013, it was proved that thermal effects prevent power increasing alot therefore, pump power value were limited in this work $[4,5]$.

In this study, two discrete differential equations were considered for laser beam and two discrete differential equation for second harmonic and one equation for harmonic population inversion for radius per $5 \mu \mathrm{m}$.

Nonlinear optics studies the phenomena on the changes and modification in optical properties of materials in the presence of light. Typically, only the laser light exhibits sufficient intensity to modify the optical features of objects [6]

\footnotetext{
* CONTACT: H_rasooli@iaut.ac.ir

DOI: http://dx.doi.org/10.28991/esj-2018-01130

(C) This is an open access article under the CC-BY license (https://creativecommons.org/licenses/by/4.0/).
} 
Frequency-doubler materials are very useful. These materials can double the frequency of the laser beam (i.e. they halve the wavelength). By this method, the output laser with $1.06 \mu$ wavelength can be converted to a radiation having $0.53 \mu$ wavelengths [7].

The laser consists of three components including active material, a pumping design and an intensifier [8].

\section{2- Theoretical Bases}

\section{2-1- The first experiment to generate the second harmonic beam in lasers}

In Figure 1, the frequency region of accessible lasers can be developed using nonlinear optical materials, such as harmonic generators and optical parameter oscillators.

The ruby laser beam at $\lambda=0.694 \mu \mathrm{m}$ wavelength is projected onto a quartz crystal and a ruby laser beam at $\lambda_{1}=0.347 \mu \mathrm{m}$ wavelength is produced. Two beams $\left(\lambda\right.$ and $\left.\lambda_{1}\right)$ are separated by a prism which are appeared on a photographic plane.

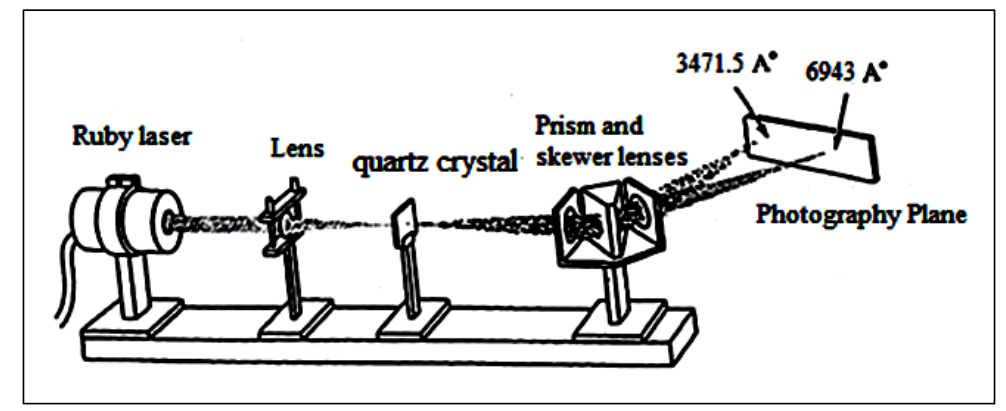

Figure 1. Generation of the second harmonic beam by nonlinear materials [1].

\section{3- Discrete Equations of Second Harmonic and Laser Beams and Inverse Population}

Forward difference for the discretization of the equations corresponding progressive fields has been used as bellow:

$$
\psi_{\mathrm{s}}^{+}(\mathrm{k}+1)=\psi_{\mathrm{s}}^{+}(\mathrm{k})+\Delta \mathrm{z}\left[-\frac{\alpha_{\mathrm{s}}}{2} \psi_{\mathrm{s}}^{+}(\mathrm{k})+\frac{\mathrm{i}}{\mathrm{L}_{1}} \psi_{1}^{+2}(\mathrm{k})\right]
$$

Where, $\psi_{s}^{+}$and $\psi_{l}^{+}$are functions of the second harmonic wave and progressive lasers, respectively. $\propto_{s}$ and Ln are the absorption coefficient of the second harmonic beam and interaction length, respectively. $\mathrm{k}$ is a variable and $\Delta \mathrm{z}$ is another variable depends on $\mathrm{z}$ (path length) and $\mathrm{k}$. also $i=\sqrt{-1}$.

$$
\psi_{1}^{+}(\mathrm{k}+1)=\psi_{1}^{+}(\mathrm{k})+\Delta \mathrm{z}\left[-\frac{\alpha_{1}}{2 \beta_{1}}+\delta \Delta \mathrm{N} \psi_{1}^{+}(\mathrm{k})+\frac{\mathrm{i}}{\mathrm{L}_{2}} \psi_{1}^{+*}(\mathrm{k}) \psi_{\mathrm{s}}^{+}(\mathrm{k})\right]
$$

In the above equation, $\Delta \mathrm{N}$ is the inverse population and $\beta_{l}$ is a variable depends on laser length.

A backward difference for discretization of the equations corresponding with retrograde fields has been used as bellow:

$$
\begin{aligned}
& \psi_{\mathrm{s}}^{-}(\mathrm{k}-1)=\psi_{\mathrm{s}}^{-}(\mathrm{k})+\Delta \mathrm{z}\left[\frac{\alpha_{\mathrm{s}}}{2} \psi_{\mathrm{s}}^{-}(\mathrm{k})-\frac{\mathrm{i}}{\mathrm{L}_{1}} \psi_{1}^{-2}(\mathrm{k})\right] \\
& \psi_{1}^{-}(\mathrm{k}+1)=\psi_{1}^{-}(\mathrm{k})+\Delta \mathrm{z}\left[-\frac{\alpha_{1}}{2 \beta_{1}}+\delta \Delta \mathrm{N} \psi_{1}^{-}(\mathrm{k})+\frac{\mathrm{i}}{\mathrm{L}_{2}} \psi_{1}^{-*}(\mathrm{k}) \psi_{\mathrm{s}}^{-}(\mathrm{k})\right]
\end{aligned}
$$

Where: $\delta$ is cross section of laser emission of $\mathrm{Nd}^{3+}$ ions and $\propto_{1}$ is absorption coefficient of the laser.

The discretization of the equation for laser level rhythm in stable status is as follows:

$$
\begin{aligned}
& \text { gg1 }=\frac{4 \eta_{\mathrm{q}} \mathrm{T}_{\mathrm{p}} \mathrm{P}_{\mathrm{p}} \alpha_{\mathrm{p}}}{h \mathrm{~W}_{\mathrm{p}} \mathrm{r}_{\mathrm{p}}^{2}} \\
& \text { gg2 }=\frac{2 \delta \mathrm{P}_{\mathrm{p}}}{\hbar \mathrm{W}_{\mathrm{l}} \mathrm{n}_{\mathrm{l}} \pi \mathrm{r}_{\mathrm{p}}^{2}}
\end{aligned}
$$


$\Delta \mathrm{N}=\left(\frac{\Delta \mathrm{N}^{0}}{\tau}+\beta_{1} \psi_{1}^{-2}+\operatorname{gg} 1 \mathrm{e}^{-\alpha_{\mathrm{p}} * \mathrm{z}}\right) /\left(\operatorname{gg} 2 \psi_{\mathrm{l}}^{+2}+\frac{1}{\tau}\right)$

Where, gg1 and gg2 are two dependent variables. Also, $\propto_{s}=193 \mathrm{~m}^{-1}, \propto_{1}=4 \mathrm{~m}^{-1}$ and $\propto_{\mathrm{p}}=340 \mathrm{~m}^{-1}$ are absorption coefficients of second harmonic beam, laser and pump, respectively [9].

$\delta=4.5 \times 10^{-19} \mathrm{~cm}^{2}$ is the cross section of laser emission of $\mathrm{Nd}^{3+}$ ions [10].

$\tau=56 \mu \mathrm{s}$ is the fluorescence lifetime [11].

$\Delta \mathrm{N}^{0}=-N_{d}$ is inverse population without pumping equaled to $N_{d}=(7 \pm 2) \times 10^{20} \mathrm{~cm}^{-3} N_{d}$ is the density of the doped elements. $d_{\text {eff }}=1.4 \times 10^{-12} \mathrm{~m} / \mathrm{v}$ is the nonlinear effective coefficient of the NYAB crystal [12].

$T_{p}=1-R_{p}=0.74$ and $P_{p}$ are passed power using the left mirror for the pump beam, and the power of the pump beam, respectively. $\eta_{\mathrm{q}}=0.26$ is quantum efficacy. $\mathrm{W}_{\mathrm{p}}$ and $\mathrm{W}_{1}$ are the angular frequency of the pump and laser beams, respectively. $\vartheta_{\mathrm{p}}, \vartheta_{\mathrm{l}}$ and $\vartheta_{\mathrm{s}}$ are the frequencies of the pump, the laser, the second harmonic beams. The reflection through the mirrors leads to $\mathrm{R}_{\mathrm{sb}}=0.03, \mathrm{R}_{\mathrm{lb}}=\mathrm{R}_{\mathrm{lf}}=\mathrm{R}_{\mathrm{sf}}=0.998$. 1 and a are the crystal length and radius. $\mathrm{I}=5 \mathrm{~mm}$ and $\mathrm{h}$ is the Hamiltonian constant [13].

$\mathrm{n}_{\mathrm{s}}=1.7050, \mathrm{n}_{\mathrm{l}}=1.7553$, are the failure coefficients of the second harmonic and the laser beams, respectively. $\mathrm{r}_{\mathrm{p}}=5 \times$ $10^{-6}$ is the beam radius [14].

$\mathrm{Z}$ is a counter or pump, $\mathrm{K}$ is a variable that belongs to $\Delta z, \Delta z=z / k, 1<\mathrm{K}<11, \mathrm{Z}=5 \mathrm{~mm}$.

$\Psi_{S}^{+}(k)$ : is forward second harmonic function.

$\Psi_{s}^{-}(k)$ : is backward second harmonic function.

$\Psi_{l}^{+}(k)$ : is forward laser wave function.

$\Psi_{l}^{-}(k)$ : is backward laser wave function.

$\Delta \mathrm{N}(\mathrm{k})$ : is population reversion rhyme.

$\eta=|\Psi|^{2}$ : is output equal to wave function as duplicated.

$\Delta \mathrm{N}=10^{24}$ : is population reversion.

$\mathrm{C}=3 \times 10^{8}$ : is light speed.

$\varepsilon_{0}=8.854 \times 10^{-12}:$ is vacancy pass constant.

$\pi=3.14$

$\lambda: \vartheta=C / \lambda$ is wavelength and $\mathrm{c}$ is light speed and $\vartheta$ is frequency.

$\mathrm{W}=2 \pi \vartheta$ is angle frequency.

$\mathrm{L}_{1}$ is interaction length No. 1 .

$L_{1}=\left(C^{3} \times n_{s}^{3} \times \varepsilon_{0} \times \pi \times r_{p}^{2}\right) /\left(w_{L}^{2} \times 2 \times d_{e f f}^{2} \times P_{p}\right)$

$\mathrm{L}_{2}$ : is interaction length of No. 2 .

$L_{2}=\left(C^{3} \times n_{L}^{2} \times n_{s} \times \varepsilon_{0} \times \pi \times r_{p}^{2}\right) /\left(w_{L}^{2} \times 2 \times d_{e f f}^{2} \times P_{p}\right)$

\section{4- Output Efficacy of Forward Second Harmonic Beam along Z-Direction for Beam Radius of $\mathrm{r}_{\mathrm{p}}=\mathbf{5} \mu \mathrm{m}$}

Figure 2 shows output of forward second harmonic beam in distance along crystal length for 20 w pump power. Output mirror (left side) has small reflection power and is a good crossing.

Output efficacy of forward second harmonic beam along $\mathrm{Z}=5 \mathrm{~mm}$ for pumping power of $\mathrm{P}_{\mathrm{p}}=20 \mathrm{~W}$ and beam radius of $r_{p}=5 \mathrm{~mm}$ is $0.634 \%$. Reflection is $\mathrm{R}_{\mathrm{SF}}=0.988$.

$$
\psi_{\mathrm{s}}^{+}(\mathrm{k}+1)=\psi_{\mathrm{s}}^{+}(\mathrm{k})+\Delta z\left[-\frac{\propto_{s}}{2} \psi_{\mathrm{s}}^{+}(k)+\frac{i}{L_{1}} \psi_{l}^{+2}(k)\right]
$$




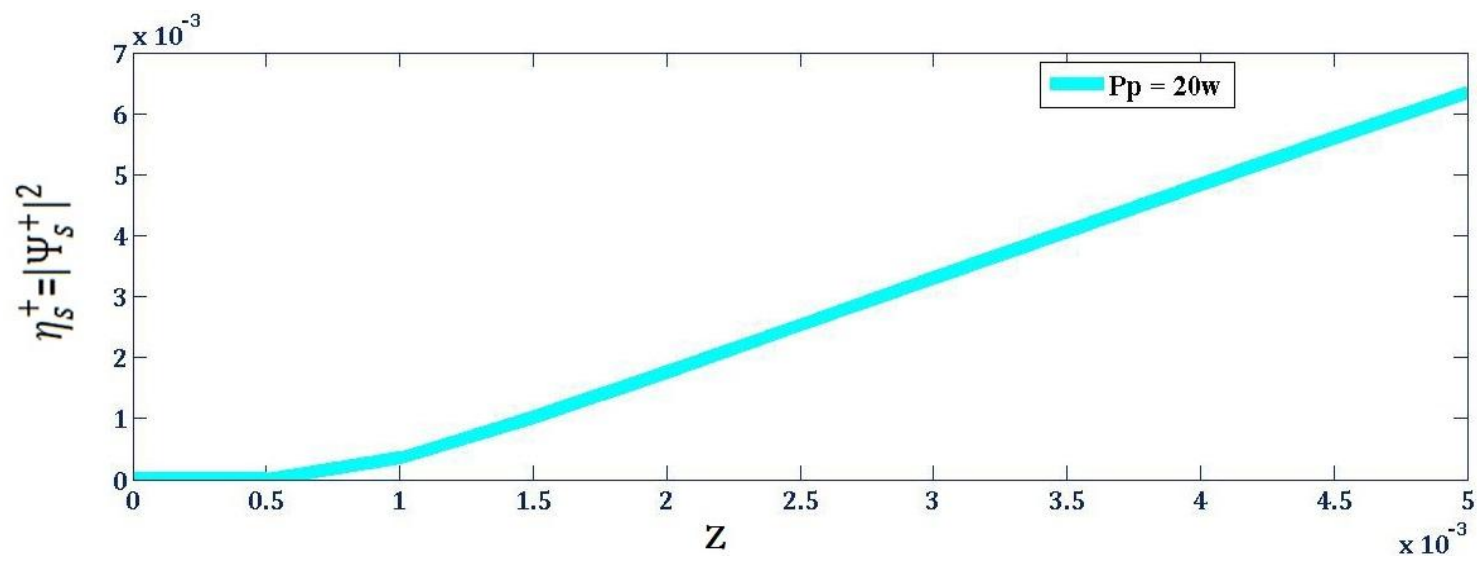

Figure 2. Efficacy of forward second harmonic beam in z-direction (path length).

\section{5- Output Efficacy of Backward Second Harmonic Beam along Z-Direction for Beam Radius of $r_{p}=5 \mu \mathrm{m}$}

Figure 3 shows that backward second harmonic beam loses some of its energy in backward (toward left side) via optical absorption in crystal (equation 7 manifests it). Backward second harmonic absorption assists in creating population reversion in laser. Increasing population backward leads to increasing infrared light $1060 \mathrm{~nm}$ which means increasing output of backward second harmonic beam.

Output efficacy of backward second harmonic beam along $\mathrm{Z}=5 \mathrm{~mm}$ for pumping power of $\mathrm{P}_{\mathrm{p}}=20 \mathrm{~W}$ and beam radius of $r_{p}=5 \mathrm{~mm}$ is $0.6327 \%$. Reflection is $R_{S b}=0.03$.

$\psi_{s}^{-}(k-1)=\psi_{s}^{-}(k)+\Delta z\left[\frac{\propto_{s}}{2} \psi_{s}^{-}(k)-\frac{i}{L_{1}} \psi_{l}^{-2}(k)\right]$

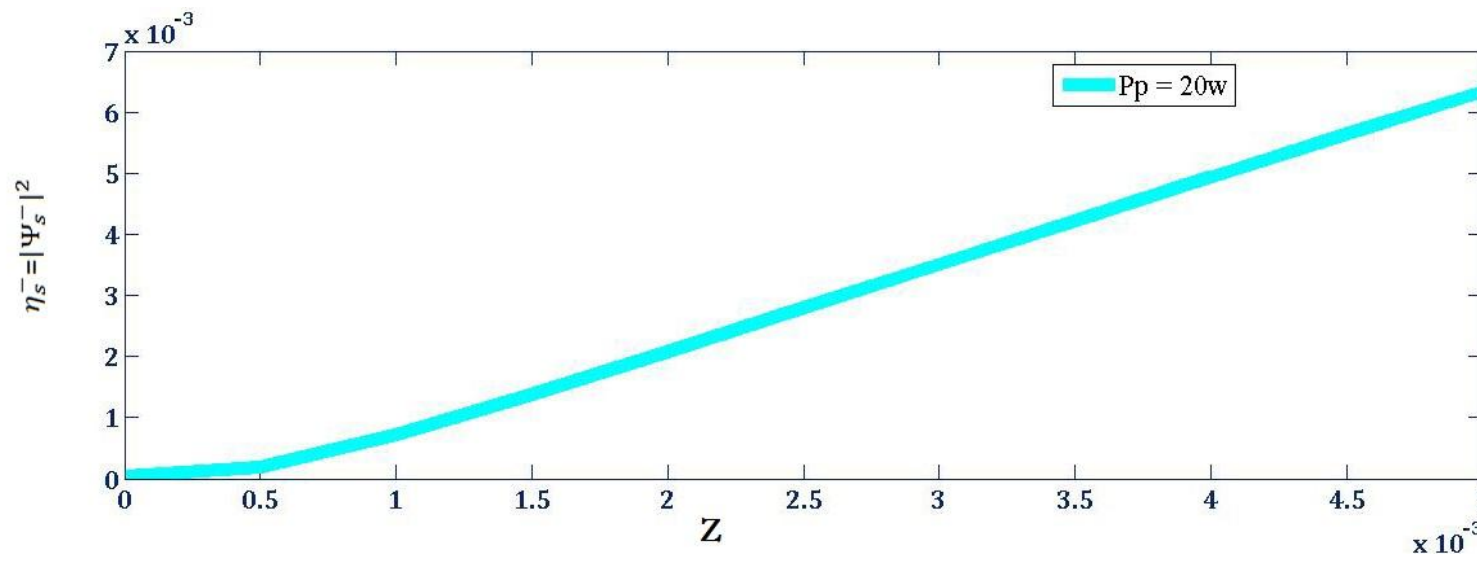

Figure 3. Efficacy of backward second harmonic beam along z-direction (path length).

\section{6- Output Efficacy of Forward Laser Beam along Z-Direction for Beam Radius of $r_{p}=\mathbf{5} \boldsymbol{\mu m}$}

Figure 4 shows output of forward laser beam. According to this figure, $20 \%$ of pumping power is transferred to laser that is expected for solid-state lasers.

Output efficacy of forward laser beam along $Z=5 \mathrm{~mm}$ for pumping power of $P_{p}=20 \mathrm{~W}$ and beam radius of $r_{p}=5 \mathrm{~mm}$ is $34.1 \%$. Reflection is $\mathrm{R}_{\mathrm{LF}}=0.988$.

$\psi_{l}^{+}(k+1)=\psi_{l}^{+}(k)+\Delta z\left[-\frac{\propto_{l}}{2 \beta_{l}}+\delta \Delta N \psi_{l}^{+}(k)+\frac{i}{L_{2}} \psi_{l}^{+*}(k) \psi_{s}^{+}(k)\right]$ 


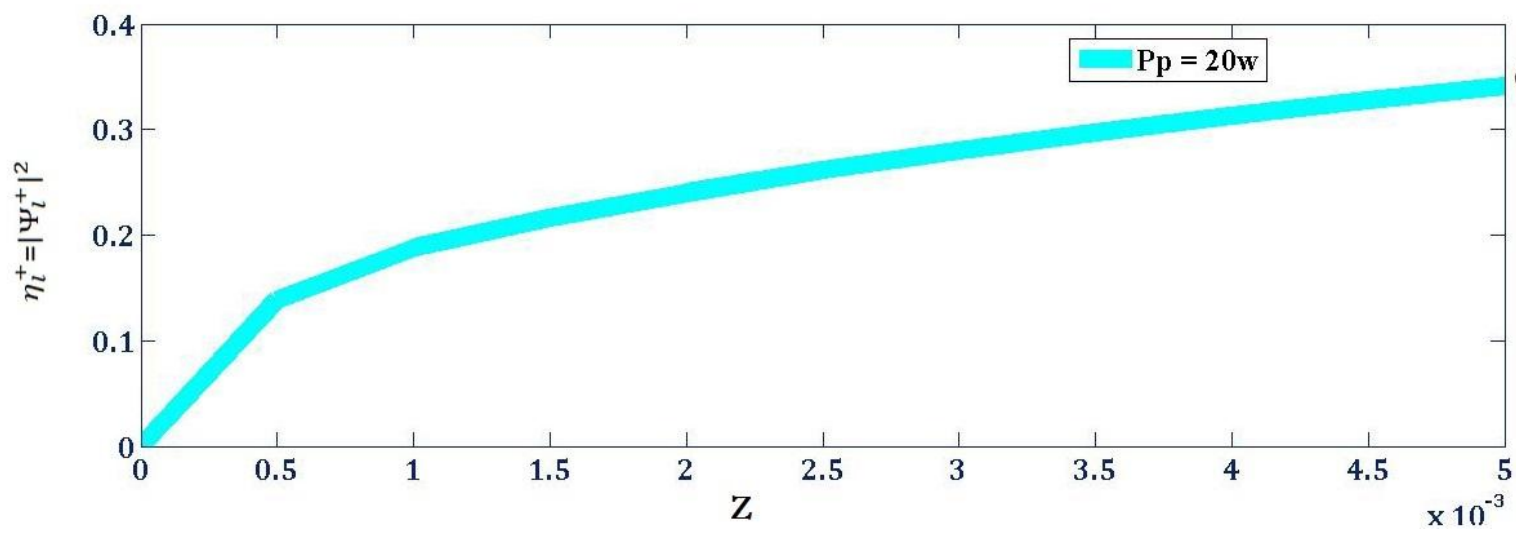

Figure 4. Output efficacy of forward laser beam along $\mathrm{z}$ direction (path length).

\section{7- Output efficacy of backward laser beam along z-direction for beam radius of $r_{p}=5 \mu \mathrm{m}$}

Figure 5 shows output of backward laser beam as $\mathrm{z}$ for $20 \mathrm{w}$ power. This figure also shows than high value of pumping power is transferred to laser beam.

Output efficacy of backward laser beam along $Z=5 \mathrm{~mm}$ for pumping power of $P_{p}=20 \mathrm{~W}$ and beam radius of $r_{p}=5 \mathrm{~mm}$ is $34.03 \%$. Reflection is $\mathrm{R}_{\mathrm{LB}}=0.998$.

$\psi_{\mathrm{l}}^{-}(\mathrm{k}+1)=\psi_{\mathrm{l}}^{-}(\mathrm{k})+\Delta \mathrm{z}\left[-\frac{\propto_{1}}{2 \beta_{\mathrm{l}}}+\delta \Delta \mathrm{N} \psi_{\mathrm{l}}^{-}(\mathrm{k})+\frac{\mathrm{i}}{\mathrm{L}_{2}} \psi_{\mathrm{l}}^{-*}(\mathrm{k}) \psi_{\mathrm{s}}^{-}(\mathrm{k})\right]$

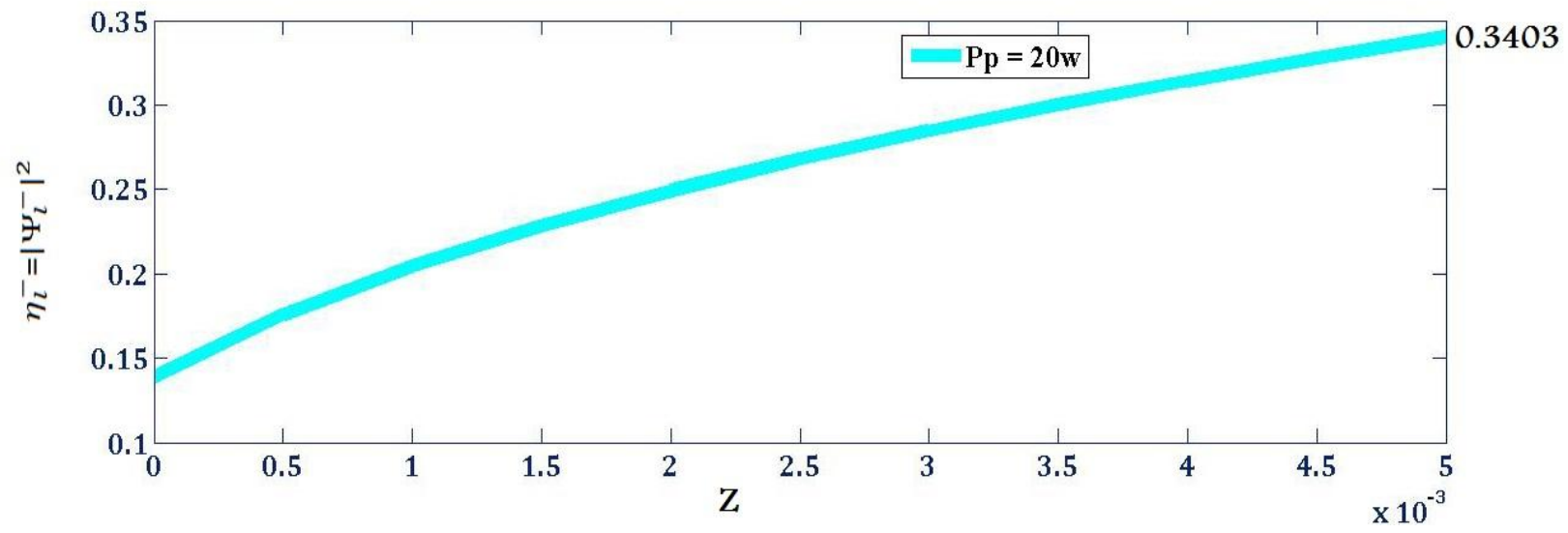

Figure 5. Output efficacy of backward laser beam in z-direction (path length).

\section{8- The Population Difference in Z-Direction for Beam Radius of $r_{p}=5 \mu \mathrm{m}$}

Figure 6 shows that population reverse depends on pumping power and has direct relationship with second harmonic beam efficacy.

The population difference along $\mathrm{Z}=5 \mathrm{~mm}$ for pumping power of $\mathrm{P}_{\mathrm{P}}=20 \mathrm{~W}$ and beam radius of $\mathbf{r}_{\mathbf{p}}=5 \mathrm{~mm}$ is 1.262 $\times 10^{24}$.

$\Delta N=\left(\frac{\Delta N^{0}}{\tau}+\beta_{l} \psi_{l}^{-2}+g g 1 * e^{-\alpha_{p} * Z}\right) /\left(g g 2 * \psi_{l}^{+2}+\frac{1}{\tau}\right)$ 


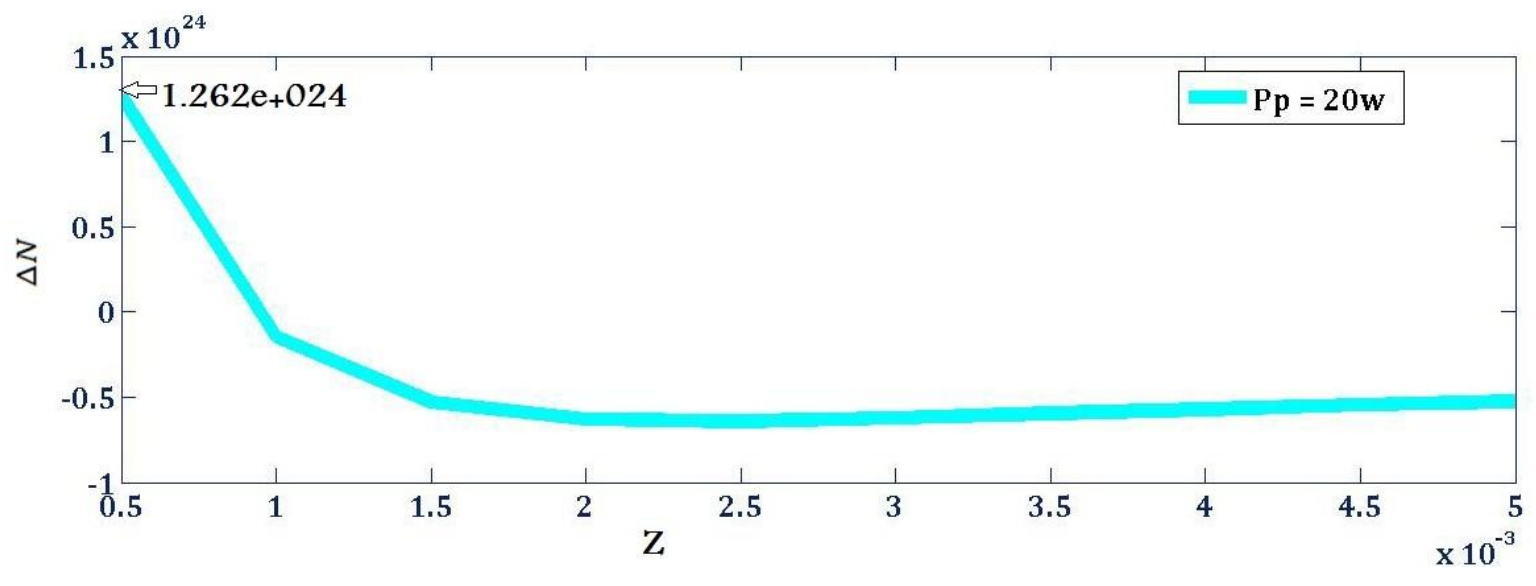

Figure 6. Population Difference along $\mathrm{Z}$ direction (path length).

\section{9- Experimental Method}

Experimental efficacy of transferring $530 \mathrm{~nm}$ beam to $1060 \mathrm{~nm}$ in NYAB crystal by Jaque et.al is as follow [15, 16]:

$$
\eta_{1060 \rightarrow 530}=\mathrm{P}_{530} / \mathrm{P}_{1060}=4.6 \mathrm{~mW} / 725 \mathrm{~mW} \approx 0.6
$$

\section{0- Conclusion}

NYAB solid-state laser is an active non-linear crystal which has been studied in recent years due to its ability in producing green light laser. High effective non-linear coefficient and high damage threshold provides the possibility of being polluted with high value of ND ion, possibility of making pumped laser with diode in green area via self doubler (SFD) NYAB crystal has been the most efficient self doubler laser.

According to computations in MATLAB, thermal effects prevents the increasing opf pumping power.

In this study, five discrete differential equations describing the conversion of $807 \mathrm{~nm}$ pump beam to $1060 \mathrm{~nm}$ laser beam and then $530 \mathrm{~nm}$ second harmonic beam have been used in Yttrium aluminum borate crystal doped with neodymium ion (the most efficient self-doubler crystal). Calculations were done in MATLAB software. Finally, the efficacies of the second harmonic and the laser beams as well as the inverse population difference increased for beam radius of $r_{p}=5 \mu \mathrm{m}$. This method is an interesting idea to achieve high efficacies in studied laser.

\section{2- References}

[1] H. Yousefi. Introduction on Solid -State Lasers and their Applications; Imam Hossein University; Vol. 1, (2002).

[2] D. Jaque, Self-frequency-sum mixing in Nd doped nonlinear crystals for laser generation in the three fundamental colours: The NYAB case, Journal of Alloys and Compounds 323-324 (2001) 204-209. https://doi.org/10.1016/s0925-8388(01)01111-2.

[3] D. Jaque, M. de la O Ramirez, L. Bausa, A. Speghlini, M. Bettinelli, and E. Cavalli, Journal of the Optical Society of America B 21 (2004) 1203-1209. https://doi.org/10.1364/josab.21.001203.

[4] M. Sabaeian, H. Nadgaran, M. De Sario, L. Mescia and F. Prudenzano, Investigation of thermal effects in octagonal double-clad fiber lasers, Optical Materials 31 (2009) 1300-1305 https://doi.org/10.1016/j.optmat.2008.10.034.

[5] A. Sayahian Jahromi, M. Sabaeian, and H. Nadgaran, Heat coupled laser rate equations: a model for Er-doped fiber lasers, Optics Communications 311 (2013) 134-139. https://doi.org/10.1016/j.optcom.2013.08.058.

[6] Boyd, Robert W. Nonlinear optics. Academic press, 2003. https://doi.org/10.1016/b978-0-12-121682-5.x5000-7.

[7] Wilson, John, and J. F. B. Hawkes. Lasers, principles and applications. Prentice Hall, 1987.

[8] Svelto, Orazio. "Principles of Lasers” (2010). doi:10.1007/978-1-4419-1302-9.

[9] Huang, Yidong, and Zundu Luo. "Relaxation Oscillation Theory for the Nd3+: YAB Self-Frequency-Doubling Laser." Optics Communications 112, no. 1-2 (November 1994): 101-108. doi:10.1016/0030-4018(94)90086-8.

[10] P. Dekker, Y. Huo, J.M. Dawes, J.A. Piper, P. Wang, B.Sh. Lu, Continuous wave and Q-switched diode-pumped neodymium, lutetium: yttrium aluminum borate lasers, Optics Communications $151 \quad$ (1998) 406-412. https://doi.org/10.1016/s00304018(98)00049-2.

[11] D. Jaque, J. Capmany, J. Rams, and J. Garcia Sole, Effects of pump heating on laser and spectroscopy properties of the 
Nd:[YAl3(BO3)4] self-frequency-doubling laser, Journal of Applied Physics $87 \quad(2000)$ 1042-1048. https://doi.org/10.1063/1.371977.

[12] H. Hemmati, Diode-pumped self-frequency-doubled neodymium yttrium aluminum borate (NYAB) laser, IEEE Journal of Quantum Electronics 28 (1992) 1169-1171. https://doi.org/10.1109/3.135243.

[13] T. Omutsu, Y. Kato, M. Shimosegawa, A. Hasegawa, I. Ogura, “Thermal effects in laser diode pumped self-frequancy-doubled NdxY1xAl3(Bo3)4 (NYAB) microchip laser" Optics Communications 118 (1995) 302-308. https://doi.org/10.1016/00304018(95)00208-p.

[14] L. Mousavi, M. Sabahian, H. Askari. Self-doubler Yttrium aluminum borate laser doped with Neodymium; Journal of Research on Many Body Systems; 4; 7; (2014): 45-54. https://doi.org/10.22055/JRMBS.2017.11591.1029.

[15] D. Jaque, et al. Continuous-wave laser properties of the self-frequency-doubling YAl3 (BO3)4: Nd crystal, Journal of the Optical Society of America B 15 (1998) 1656-1661. https://doi.org/10.1364/josab.15.001656.

[16] J. Bartschke, R. Knappe, K.J. Boller, and R. Wallenstrin, Investigation of efficient self-frequency-doubling Nd: YAB lasers, IEEE Journal of Quantum Electronics 33 (1997) 2295-2230. https://doi.org/10.1109/3.644113. 